\title{
THALAMIC DEGENERATION FOLLOWING BILATERAL PREMOTOR FRONTAL LOBE ATROPHY OF THE STRÜMPELL TYPE
}

\author{
BY
}

\section{R. M. NORMAN}

(From the Burden Mental Research Department, Stoke Park Colony, Bristol)

(RECEIVED 20TH OCTOBER, 1945)

THE case described in this paper confirms and amplifies an observation made by Stern (1942) concerning thalamo-frontal projection in man. Stern described a traumatic lesion involving the prefrontal and premotor areas of the cerebral cortex associated with severe retrograde degeneration of the dorso-medial and, unexpectedly, of the medial third of the anterior part of the ventro-lateral thalamic nuclei. The novelty of the latter finding lay in the apparently greatly increased premotor projection field of this nucleus in man as compared with other primates, since it is known that in monkeys the anterior part of the ventro-lateral nucleus projects mainly upon the percentral gyrus (Walker, 1938a). Human material suitable for anatomical analysis of this sort is rare and since the present case has additional features of pathological interest it seems to be worthy of record.

\section{Case Report}

J. C., a female, was admitted to Stoke Park Colony for mental defectives when 8 years 6 months old and died aged 18 years and 8 months.

History prior to Admission.-Birth was normal and no anxiety as to the infant's progress was experienced by the parents until the age of 13 months when she had an illness characterized by constipation, vomiting, and later by coma lasting 3 days. After this the patient lost the power of walking, and severe mental defect developed. Nevertheless, she soon learned to walk again. At the age of 5 years she began to suffer from epilepsy and was reported to be sleeping badly at night and to be very difficult to manage. An epileptic attack which was witnessed consisted in a sudden blankness with twitching of the arms and legs only. When 6 years old she was admitted to the National Hospital, Queen Square, under the care of Dr. Hinds Howell. The records of the hospital provide the following notes as to her condition at this time: "On examination there was gross mental deficiency. She kept uttering weird shrieks and was unable to speak or recognize anything. Examination was necessarily difficult, but what could be carried out showed no gross abnormality of the nervous system. During her stay in the hospital she was quite uncontrollable ".

Examination after Admission.-The weight and stature were normal for her age of $8 \frac{1}{2}$ years. Using Smedley's dynamometer she recorded normal right and left grips of 12 and 10 kilograms respectively. Her mental age was only 2 years and 5 months as estimated by the Merrill-Palmer scale of tests. She was able to cut paper with scissors, put 16 cubes into a box, and fit the square Wallin pegs into their board within the time intervals appropriate to normal three-year-olds. She was able to walk and to stand on one leg without overbalancing. There was no spontaneous speech but she could repeat a few simple words. No gross abnormality was found in examination of the nervous system. Both plantar reflexes were flexor but the knee jerks were abnormally brisk. The left optic disc was pale with small arteries. The blood Wassermann reaction was negative. The patient suffered from frequent epileptic fits which were not fully controlled by gardenal gr.i.b.d. She was occasionally noisy but during her stay in the Colony her behaviour did not differ markedly from that of other epileptics of low mental calibre. Death occurred in her nineteenth year from pulmonary tuberculosis.

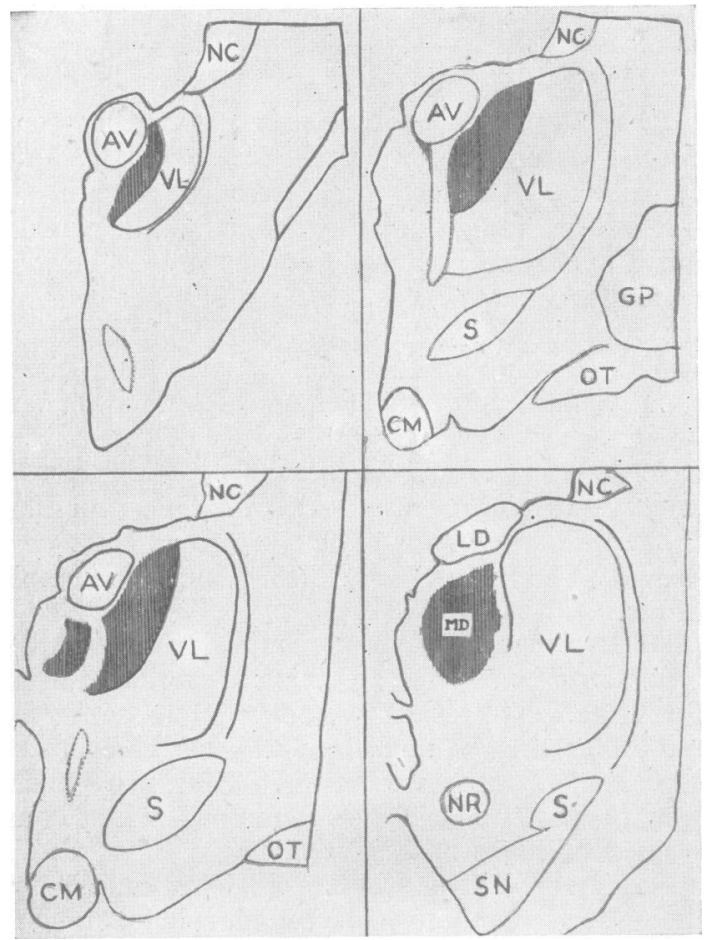

FIG. 1.-Four representative coronal sections of the right thalamus, showing the extent of retrograde degeneration in the dorso-medial and ventrolateral nuclei.

Abbreviations: AV, nucleus antero-ventralis; $\mathrm{CM}$, corpus mammillare; GP, globus pallidus; LD, nucleus lateralis dorsalis; MD, nucleus medialis dorsalis; NC, nucleus candatus; NR, nucleus ruber; OT, tractus opticus; S, corpus subthalamicum; SN, substantia nigra. 

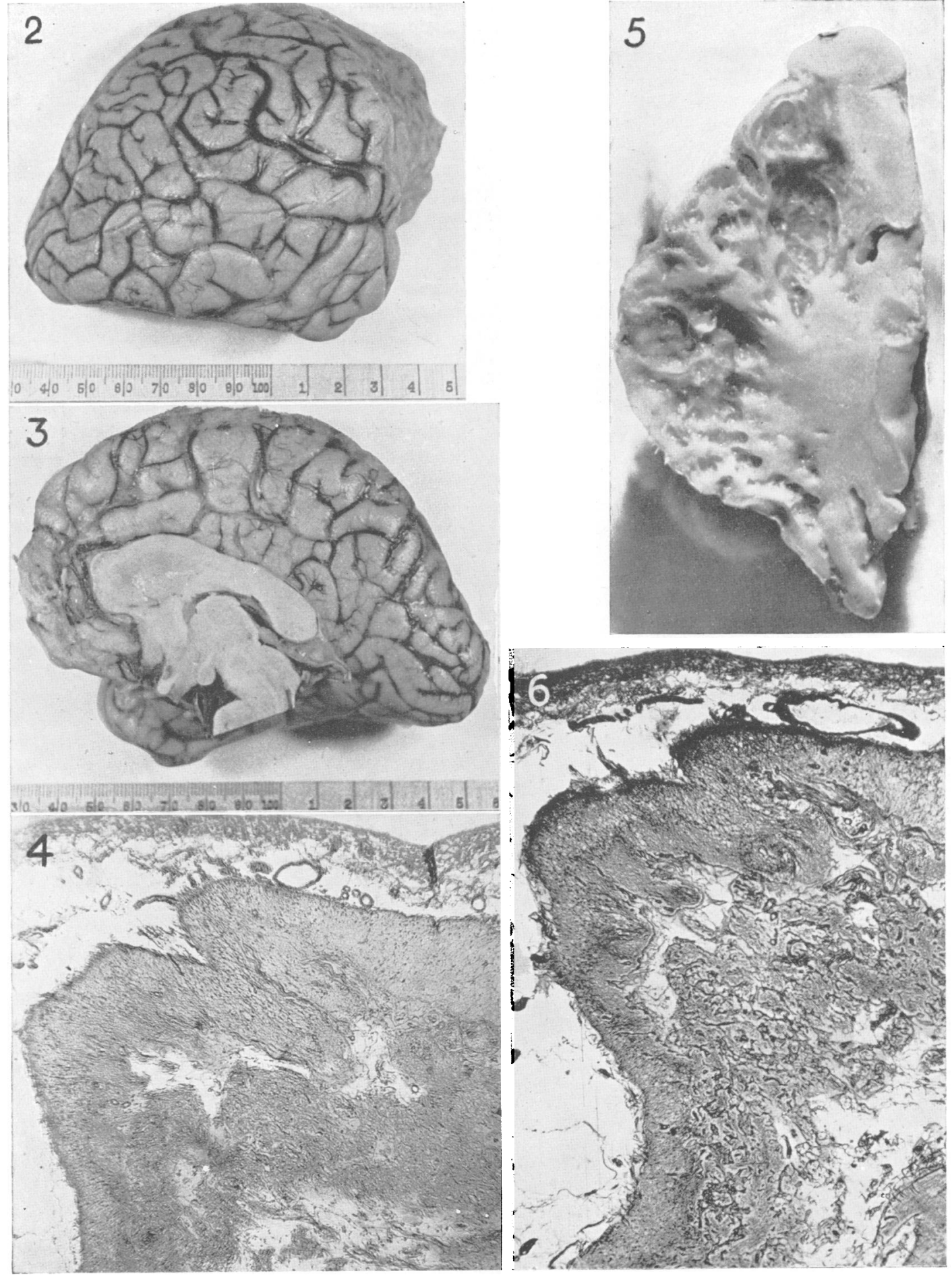

Fig. 2.-Right cerebral hemisphere, showing atrophy of the front lobe anterior to the precentral gyrus.

FIG. 3.-Right cerebral hemisphere, medial surface, showing preservation of part of the superior frontal and cingulate gyri.

FIG. 4.-A representative section from the atrophied frontal cortex, showing the thickened leptomeninges, gliosis and cavitation of the brain substance. Mallory's phosphotungstic acid hæmatoxylin. $\times 48$.

FIG. 5.-A coronal section through the atrophied right frontal lobe, showing multiple cavitation and relative preservation of the superior frontal gyrus and gyrus rectus. $\times 1 \cdot 5$.

Fig. 6.-The same cortical area as in Fig. 4. Note the thickening and crowding together of the blood vessels. Gomer reticulin stain. $\times 48$. 


\section{Pathological Examination of the Central Nervous} System

Macroscopic Examination.-The brain was small and, after fixation, weighed only $765 \mathrm{gm}$., of which cerebellum and brain-stem accounted for $122 \mathrm{gm}$.

The frontal lobes, exclusive of the precentral and small portions of the superior frontal gyri appeared to be symmetrically atrophied, the convolutions being flattened, unduly firm to the touch, and covered by thickened and opaque leptomeninges. This reduction in volume was remarkable, the frontal lobes scarcely projecting beyond the level of the temporal poles (Figs. 2 and 3). The lengths of the right and left central sulci as measured by a thread were respectively 9.1 and $9.8 \mathrm{~cm}$. Both precentral gyri appeared intact. On the left side about $2 \mathrm{~cm}$. and on the right side about $5 \mathrm{~cm}$. of the posterior part of the superior frontal gyrus were preserved in the form of triangular wedges of cortex, the apices of which pointed forward. On the medial surfaces of the hemispheres the cingulate gyri were preserved as far anteriorly as the genu of the corpus callosum, which itself showed shrinkage and rarefaction at this point. On the inferior surface of the frontal lobes only the crowns and medial walls of the gyri recti appeared intact.

On section, the shrunken part of the brain showed a sponge-like appearance of multiple small cavities, all distinction between grey and white matter being lost (Fig. 5). The lateral wall of the right superior frontal gyrus was seen to be eroded by the destructive process. The parietal, temporal, and occipital lobes appeared normal.

Microscopic Examination.-The leptomeninges covering the shrunken gyri showed marked fibrosis and were adherent to the subjacent cortex. The atrophied part of the frontal lobe presented a remarkably uniform appearance, being devoid of myelin and composed of a coarse network of piloid neuroglia (Fig. 4). Owing to the shrinkage of the tissue, the blood vessels appeared to be more numerous than usual and their adventitial coats were seen to be somewhat thickened (Fig. 6). Beneath a superficial rind of cortex, itself the seat of intense gliosis, multiple cavities and rarefactions extended throughout the whole depth of the frontal lobe. Normal nerve cells were absent but large aggregations of "calcified" cells and débris were commonly encountered.

The Extent of the Intact Frontal Cortex.-Six blocks of tissue were examined from each precentral gyrus. On the right side the nerve cell and myelin preparations were normal except in the inferior centimetre of the gyrus where the anterior wall of grey matter was atrophied, the crown, posterior wall and most of the central core being intact. On the left side the lowest $3 \mathrm{~cm}$. of the precentral gyrus were completely atrophied and undercut by the pathological process.

As regards the superior frontal gyrus of the right hemisphere, the microscopically preserved part included about $3 \mathrm{~cm}$. of agranular cortex, the fifth layer of which contained scattered Betz cells.
The Thalami.-Coronal sections stained to demonstrate nerve cells and myelin were prepared from ten different levels of each thalamus. The extent of retrograde degeneration in the right thalamus was also followed in incomplete serial sections stained both by toluidine blue and by Anderson's modification of the Kultschitsky-Pal method, sections being taken at $100 \mu$ intervals. No significant difference was found between the two sides and since the right precentral gyrus had been shown to be virtually intact the condition of the right thalamus was studied in greater detail and is illustrated in the accompanying drawings (Fig. 1) and photographs (Figs. 7-13). The dorso-medial nucleus showed complete degeneration. On the other hand, the nuclei of the midline, the parataenial and paraventricular cell groups and also the cell groups occupying the position of the internal medullary lamella were fully preserved and, indeed, owing to the small size of the thalamus as a whole, appeared relatively prominent. In sections taken from the most rostral part of the thalamus the anterior ventral nucleus, recognized by the coarse clumping of the nerve cells, appeared to be intact. In the ventro-lateral nucleus, nerve cells were either absent or grossly reduced in number in a medial strip which occupied about one-third of the transverse width of the nucleus and extended from the anterior limit of the ventro-lateral cell group to a plane passing through the level of the rostral extremity of the red nucleus. Another small area of degeneration was seen to occupy the position of the anteromedial nucleus of lower animals, that is to say, it lay on the ventral aspect of the main antero-ventral nucleus medial to the entrance of the bundle of Vicq d'Azyr. The areas of degeneration showed marked gliosis and often contained "calcified" nerve cells. In sections stained for myelin a conspicuous pallor was noted in these areas, especially in the affected position of the ventro-lateral nucleus, owing to the absence of the numerous closely packed bundles of fibres which are normally present.

Other parts of the Brain.-No pathological changes were encountered in representative blocks of tissue taken from parietal, occipital, and temporal lobes (including hippocampus) of both hemispheres. The putamen, globus pallidus and subthalamus were also intact on both sides, as were pons and medulla. The medullary pyramids (Fig. 14), though rather small, were fully myelinated and showed no abnormal gliosis.

The olfactory tracts showed a mild central gliosis but their myelinated fibres were preserved. The anterior cerebral vessels also appeared normal.

\section{Comment}

The examination detailed above has shown that the whole of the precentral gyrus, together with the posterior portion of the superior frontal gyrus, were intact in the right frontal lobe. In terms of cytoarchitectonics these preserved areas comprise the whole of Area 4 and the major part of Area 6 (Brodmann, 1925), or the whole of Area F.A. and 

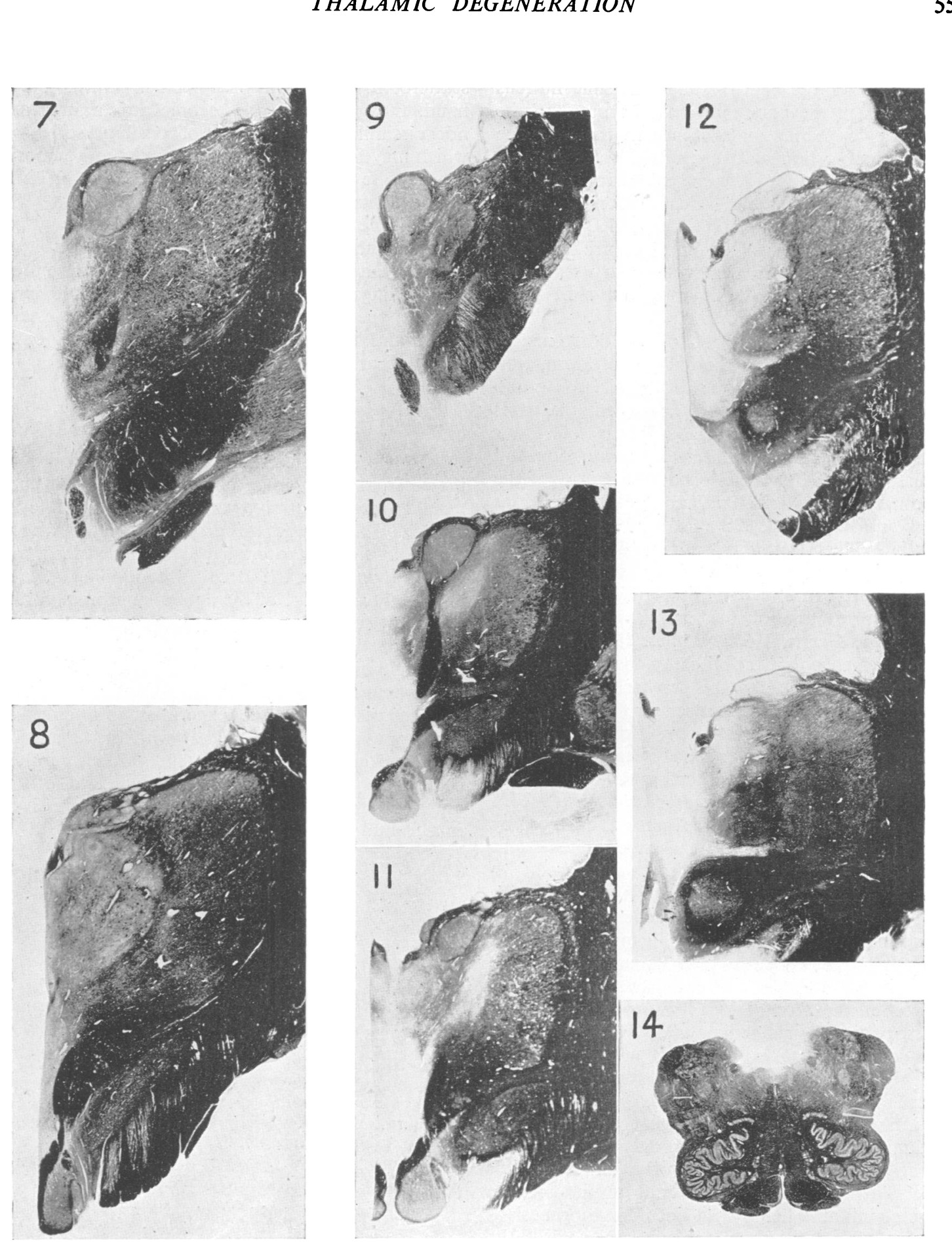

FIGs. 7 and 8.-Coronal sections through a normal adult thalamus. Kultschitsky-Pal stain (Anderson's modification). $\times 2$.

Figs. 9-13.-Coronal sections through the right thalamus. Note in Figs. 10 and 11 the pallor of the medial part of the ventro-lateral nucleus and in Figs. 12 and 13 the pallor of the dorso-medial nucleus. Stain and magnification as in Figs. 7 and 8.

Fig. 14.-Medulla, showing intact pyramidal tracts. Kultschitsky-Pal stain. $\times 2 \cdot 2$. 
the major part of Area F.B. (von Economo, 1929). It would seem, therefore, that in man the anteromedial part of the ventro-lateral thalamic nucleus projects upon the cortex in a field frontal to the electrically excitable zone. Since in the macaque the anterior portion of the ventro-lateral cell mass projects mainly upon Area 4, while in the chimpanzee both Area 4 and Area 6 receive efferent fibres from this nucleus (Walker, 1938b), it is evident that with the expansion of the frontal lobes associated with rise in the evolutionary scale, the projection of this thalamic radiation shifts progressively in a frontal direction.

The complete degeneration of the dorso-medial nucleus is in contrast to the findings of Stern, who described in his case a preserved medial cell group of considerable extent and suggested that this represented the pars magnocellularis of lower animal forms. It was formerly considered doubtful whether this medial large-celled component of the dorso-medial nucleus had cortical connections (Clark and Boggon, 1935; Walker, 1936), but more recently Walker (1940) has shown that in macaca efferent fibres pass to the orbital surface of the frontal lobe. In the cat, also, the pars magnocellularis projects upon the granular frontal cortex (Waller and Barris, 1937; Waller, 1940). In man there is evidence that the whole of the dorso-medial nucleus is connected to the cerebral cortex, since total degeneration had occurred in the example of infantile hemiplegia reported by Clark and Russell (1940). A similar (unpublished) case of my own confirms their findings in every detail. It thus appears probable that the preservation of a media component of the dorso-medial nucleus observed by Stern is to be correlated with the more limited extent of the cortical lesion in his case as compared with mine in which the orbital surface of the frontal lobe was widely affected. The degeneration of the antero-medial thalamic nucleus in my case may be similarly explained since it is known (in macaca) that this cell group relays impulses to the inferior surface of the frontal lobes and possibly to the anterior part of the cingulate gyrus (Walker, 1938a).

In conclusion, a few points of pathological and clinical interest may briefly be mentioned. The abrupt onset of the illness, its serious effect upon the infant's intelligence, and the character of the residual lesions are features strongly reminiscent of the so-called "encephalitis" of Strümpell. This variety of encephalopathy is not an uncommon cause of infantile hemiplegia but its pathogenesis is obscure. There is abundant evidence against the early view that the condition represents a cerebral variety of anterior polio-myelitis (Rothman, 1931). In the present case, moreover, the bilateral symmetry and sharp demarcation of the atrophied frontal cortex would be difficult to reconcile with the widely scattered foci of a virus encephalitis. On the other hand, the gross and microscopic appearances are not dissimilar to the form of cerebral softening which may follow phlebostasis or phlebothrombosis in early life and which is often the sequel of birth injury (Marburg and Casamajor, 1944). The resemblance between the pathological findings in traumatic softening of this type and that associated with Strümpell's encephalopathy has been noted by Dollinger (1927), but a causal relationship between the two conditions is certainly not established and there is nothing to suggest that birth injury occurred in the present case.

\section{Summary}

In the brain of an epileptic imbecile atrophy of the cerebral cortex involved the major part of both frontal lobes exclusive of the percentral gyri. Clinically there was no gross locomotor defect. The right side of the brain was examined in detail. It was found that the Betz-cell containing Area 4 and the greater part of the agranular frontal cortex (Area 6) were intact. In the thalamus severe retrograde degeneration implicated the whole of the dorso-medial nucleus and also the medial third of the anterior part of the ventro-lateral nucleus.

Comparison with the experimental findings in monkey and ape suggests that with the expansion of the frontal lobes associated with rise in the evolutionary scale so the projection field of the anterior ventro-lateral nucleus shifts progressively in a frontal direction.

\section{REFERENCES}

Brodmann, K. (1925). Vergleichende Localisationslehre der Grosshirnrinde. 2nd. Ed. Leipzig.

Clark, W. E. Le Gros, and Boggon, R. H. (1935). Phil. Trans. roy. Soc. Lond., Series B, 224, 313

_ and Russell, D. S. (1940). J. Neurol. Psychiat., n.s. 3, 123 .

Dollinger, A. (1927). Ergebn. inn. Med. Kinderheilk., 31, 373.

Economo, C. von (1929). The Cytoarchitectonics of the Human Cerebral Cortex. London.

Marburg, O., and Casamajor, L. (1944). Arch. Neurol. Psychiat. (Chicago), 52, 170.

Rothman, P. E. (1931). Amer. J. dis. Child, 41, 124.

Stern, K. (1942). J. Anat., 76, 302.

Walker, A. E. (1936). J. comp. Neurol., 64, 1. (1938a). The Primate Thalamus. Chicago. (1938b). J. Anat., 73, 37.

- (1940). J. comp. Neurol., 73, 87.

Waller, W. H., and Barris, R. W. (1937). Ibid., 67, 317. (1940). Ibid., 73, 117. 